\title{
Zeta regularization of infinite products
}

\author{
Mauro Spreafico ${ }^{1,2 *}$ \\ ${ }^{1}$ Dipartimento di Matematica e Fisica, Università del Salento, Lecce, Italy \\ 2 ICMC, Universidade de São Paulo, São Carlos, Brazil
}

\section{Edited by:}

Valter Moretti, Università degli Studi

di Trento, Italy

Reviewed by:

Valter Moretti, Università degli Studi

di Trento, Italy

Sonia Mazzucchi, Università degli

Studi di Trento, Italy

*Correspondence:

Mauro Spreafico, ICMC,

Universidade Sào Paulo, Sào Carlos,

13566-590, Brazil

e-mail: mauro.spreafico@

unisalento.it; mauros@icmc.usp.br
We describe the procedure of zeta regularization of an infinite product, in particular following the technique we introduced in previous works (13).

Keywords: zeta functions, regularized products, functional determinants, $z, z z$
The problem of regularizing infinite products is a long standing one in mathematical and theoretical physics, as well as in mathematics, of course. Several either formal or rigorous techniques have been introduced. One that obtained an undiscussed favor is the zeta regularization. The idea is simple to write down in a formal way (so as it as been used for a long times by physicists), and has got a rigorous mathematical formulation. This produced a huge number of interesting (and uninteresting) applications, with many useful (and non-useful) results. In this notes, we briefly recall the main features of the zeta regularization procedure, in particular following the approach introduced in our works.

Given a sequence $S=\left\{a_{n}\right\}_{n=1}^{\infty}$ of non-vanishing complex numbers, arranged by increasing module, the formal product

$$
\prod_{n=1}^{\infty} a_{n}
$$

appears in the series of formal equalities

$$
\log \prod_{n=1}^{\infty} a_{n}=\sum_{n=1}^{\infty} \log a_{n}=-\left.\frac{\mathrm{d}}{\mathrm{d} s} \sum_{n=1}^{\infty} a_{n}^{-s}\right|_{s=0}
$$

This suggests to call zeta regularized product of the $a_{n}$, the number

$$
\mathrm{e}^{-\left.\frac{\mathrm{d}}{\mathrm{d} s} \sum_{n=1}^{\infty} a_{n}^{-s}\right|_{s=0}}
$$

In particular, if $S$ is either the sequence of the eigenvalues of a matrix $A$, or the discrete spectrum of a differential operator $A$, we call zeta regularized determinant of $A$ the number $\operatorname{det}_{\zeta} A \operatorname{defined}$ by

$$
\log \operatorname{det}_{\zeta} A=-\left.\frac{\mathrm{d}}{\mathrm{d} s} \sum_{n=1}^{\infty} a_{n}^{-s}\right|_{s=0} .
$$

This formal approach is based on the possibility of definining the function of the (complex) variable $s$ :

$$
\zeta(s, S)=\sum_{n=1}^{\infty} a_{n}^{-s},
$$

called the zeta function associated to the sequence $S$, and on the analytic properties of it. In particular, $\zeta(s, S)$ should be defined at $s=0$ with its first derivative. As a matter of fact, it is not strictly necessary that $\zeta(s, S)$ is defined at $s=0$ : it is sufficient that there exists a Lauren-Mac Laurin series for it.

Zeta functions have been deeply investigated in mathematics, mainly in analysis and number theory, and this study provided several different types of sequences for which the zeta regularization process works, and therefore possible applications to physics. In general, the series definition of the zeta function is analytic in a region of the complex plane that does not contain the origin, so analytic continuation is necessary. The problem is thus the one of finding out a suitable representation of the zeta function, that permits to describe its analytic continuation. The natural example is the Riemann zeta function

$$
\zeta_{\mathrm{R}}(s)=\sum_{n=1}^{\infty} n^{-s},
$$

where the sequence is $S=\{n\}_{n=1}^{\infty}$. As well known, we have the Hermite formula

$$
\zeta_{\mathrm{R}}(s)=\frac{1}{2}+\frac{1}{s-1}+2 \int_{0}^{\infty} \frac{\left(1+t^{2}\right)^{-\frac{s}{2}} \sin (s \arctan t)}{\mathrm{e}^{2 \pi t}-1} d t,
$$

that extends analytically in the whole complex plane up to the simple pole at $s=1$ (with residue 1). The determination of the value of $\zeta_{R}^{\prime}(0)$ is a bit harder, but follows as well differentiating the 
formula of Hermite (and recalling some properties of the Euler Gamma function).

A main class of sequences that admit a zeta regularization is given by the spectrum of a class of linear operators: more precisely, given a non negative self adjoint differential operator $H$ on a compact manifold, one can prove that the zeta function associated to the spectrum of $H$ has an analytic extension at $s=0$, and (hopefully) compute the derivative. Following Hawking (4), this provides a first important application in quantum physics, to deal with gaussian path integrals. The partition function for a finite temperature quantum field theory on a ultrastatic spacetime with compact spazial section is constructed as follows. Let $M$ be a compact Riemannian manifold of dimension $n$, and consider the product $N=S_{r}^{1} \times M$, where $S_{r}^{1}$ is the circle of radius $r=\frac{\beta}{2 \pi}$, and $\beta=\frac{1}{T}$ is the inverse of the temperature. Let $L$ be some non-negative self adjoint operator (typically the Laplacian) acting on some functions space (we shall deal with scalar fields) defined on $M$ and $H=-\partial_{u}^{2}+L$. The canonical partition function at temperature $T$ of this model may be formally written as

$$
Z=\operatorname{det}^{-\frac{1}{2}}\left(\ell^{2} H\right)
$$

where $\ell$ is some renormalization constant. The zeta function of $H$ is defined by (where $\mathrm{Sp}_{+} H$ denotes the positive part of the spectrum of $H$ )

$$
\zeta(s ; H)=\sum_{\lambda \in \mathrm{Sp}_{+} H} \lambda^{-s},
$$

when $\operatorname{Re}(s)>s_{0}$ (for some suitable $s_{0}$ ), the regularized functional determinant of $H$ is defined by

$$
\operatorname{det}_{\zeta} H=\left.\mathrm{e}^{-\frac{\mathrm{d}}{\mathrm{d} s} \zeta(s ; H)}\right|_{s=0}
$$

and the partition function is then

$$
\log Z=\frac{1}{2} \zeta^{\prime}(0 ; H)-\frac{1}{2} \zeta(0 ; H) \log \ell^{2}
$$

If the underlying manifold is not compact, there could be a continuous spectrum. In such a case, the idea of relative zeta function can be used (8). This works as follows. Let $\left(H, H_{0}\right)$ be a pair of non negative self adjoint linear operators such that the difference of the resolvents (or the difference of the heat operators) is of trace class (some further properties are necessary, see for example 16 or 1 for details). Then, the relative zeta function is defined using the Mellin transform

$$
\zeta\left(s ; H, H_{0}\right)=\frac{1}{\Gamma(s)} \int_{0}^{\infty} t^{s-1} \operatorname{Tr}\left(\mathrm{e}^{-t H}-\mathrm{e}^{-t H_{0}}\right) \mathrm{d} t,
$$

and the partition function reads

$$
\log Z=\frac{1}{2} \zeta^{\prime}\left(0 ; H, H_{0}\right)-\frac{1}{2} \zeta\left(0 ; H, H_{0}\right) \log \ell^{2} .
$$

While several rigorous approaches have been introduced and developed in order to investigate the analytic properties of the zeta function, comparatively few attempts to deal with the zeta determinant can be found in the literature. We briefly recall here our technique (see 12-14), whose main characterization is the fact of using a "new" spectral function, called Gamma function, instead of the usual heat kernel.

Let $S=\left\{a_{n}\right\}_{n=1}^{\infty}$ be a sequence of non vanishing complex numbers with unique accumulation point at infinity, ordered by increasing modules. Assume the exponent of convergence of $S$ is finite $\left(e(S)=\limsup _{n \rightarrow \infty} \frac{\log n}{\log \left|\lambda_{n}\right|}<\infty\right)$. We define the Gamma function associated to $S$ by the convergent Weierstrass canonical product

$$
\frac{1}{\Gamma(-\lambda, S)}=\prod_{n=1}^{\infty}\left(1+\frac{-\lambda}{a_{n}}\right) \mathrm{e}^{\sum_{j=1}^{p} \frac{(-1)^{j}}{j} \frac{(-\lambda)^{j}}{a_{n}^{j}}} .
$$

where $p$ is the genus of $S$, i.e. the least integer $p$ such that the series $\sum_{n=1}^{\infty} a_{n}^{-p-1}$ converges (absolutely).

We have the following formulas, relating the Gamma function to the other relevant spectral functions:

$$
\begin{aligned}
f(t, S)-1 & =-\frac{t}{2 \pi i} \int_{\Lambda_{\theta, c}} \mathrm{e}^{-\lambda t} \log \Gamma(-\lambda, S) \mathrm{d} \lambda, \\
\zeta(s, S) & =\frac{s}{\Gamma(s)} \int_{0}^{\infty} t^{s-1} \frac{1}{2 \pi i} \int_{\Lambda_{\theta, c}} \frac{\mathrm{e}^{-\lambda t}}{-\lambda} \log \Gamma(-\lambda, S) \mathrm{d} \lambda \mathrm{d} t,
\end{aligned}
$$

where $\quad \Lambda_{\theta, c}=\left\{z \in \mathbb{C}|| \arg (z-c) \mid=\frac{\theta}{2}\right\}$, oriented counter clockwise, and

$$
f(t, S)=\sum_{n=1}^{\infty} \mathrm{e}^{-t a_{n}}+1
$$

is the heat function, i.e. the trace of the heat operator.

In the classical approach the heat function is used to study the analytic properties of the zeta function using the Mellin transform

$$
\zeta(s, S)=\frac{1}{\Gamma(s)} \int_{0}^{\infty} t^{s-1}(f(t, S)-1) \mathrm{d} t .
$$

In fact, by this formula, explicit knowledge of the asymptotic expansion of the heat function for small $t$ gives the position of the poles of the zeta function and the values of the residues.

In our approach, the main spectral function is the logarithmic Gamma function. It is clear, by the formula in Equation (1), that the asymptotic expansion of the heat function for small $t$ follows from the one of the logarithmic Gamma function for large $\lambda$. Therefore, all information contained in the heat function are contained in the Gamma function (13).

However the main improvement obtained using the Gamma function is due to the fact that the coefficients in the asymptotic expansion of the logarithm of the Gamma function for large $\lambda$ give the derivative of the zeta function [using the formula in Equation (2)], while this information is not available from the coefficients of the expansion of the heat function. In other word, 
the expansion of the Gamma function is a global invariant, as the derivative of the zeta function, while the expansion of the heat function, as well known, is local, namely can be determined using local quantities constructed using the geometry of the manifold. This is the content of the next theorem (see 13 or the first section of 15 for details and proofs).

Theorem 0.1. If $S$ is a regular sequence of spectral type of order $\alpha_{N} \leq 0$, then the associated zeta function is regular at $s=0$, and near $s=0$

$$
\zeta(s, S)=-a_{0,1}-a_{0,0} s+O\left(s^{2}\right),
$$

where the $a_{0,1}$ and $a_{0,0}$ are the coefficients of the logarithmic term and of the constant term in the asymptotic expansion of the logarithmic Gamma function for large $\lambda$, namely

$$
\begin{aligned}
\log \Gamma(-\lambda, S)= & \sum_{j=0}^{N} a_{\alpha_{j}, 0}(-\lambda)^{\alpha_{j}}+\sum_{j=0}^{N} a_{\alpha_{j}, 1}(-\lambda)^{\alpha_{j}} \log (-\lambda) \\
& +O\left((-\lambda)^{\alpha_{N}}\right) .
\end{aligned}
$$

In the example of the Riemann zeta function considered above, the Gamma function is

$$
\begin{aligned}
\log \Gamma(-\lambda, S) & =-\log \prod_{n=1}^{\infty}\left(1+\frac{-\lambda}{n}\right) \mathrm{e}^{-\frac{-\lambda}{n}} \\
& =\gamma(-\lambda)+\ln \Gamma(-\lambda+1)
\end{aligned}
$$

that has the following expansion for large $\lambda$

$$
\begin{aligned}
& \log \Gamma(-\lambda, S)=(-\lambda) \ln (-\lambda)+(\gamma-1)(-\lambda)+\frac{1}{2} \ln (-\lambda)+\frac{1}{2} \\
& \ln 2 \pi+\sum_{j=1}^{\infty} \frac{B_{2 j}}{2 j(2 j-1)}(-\lambda)^{1-2 j} .
\end{aligned}
$$

\section{Application of Theorem 0.1 gives}

$$
\zeta_{R}(0)=-\frac{1}{2}, \quad \zeta_{R}^{\prime}(0)=-\frac{1}{2} \log 2 \pi
$$

We conclude with a remark on references. It is impossible to mention all the relevant references in this field. We decided to cite explicitly only our works (that contains the details on the approach that we briefly outlined here), and some particular references that are the undiscussed references usually associated to some particular subject. However, we want to add here some further references that are either interesting works on some particular problem or that contain themselves a good list of references on some aspect of the field. For rigorous treatment of regularized products we suggest $(5-7,17)$. For a good account of applications to physics see (2). For heat kernel methods and functional determinant see $(3,10)$. Of course these suggestions are far from being exhaustive.

\section{REFERENCES}

1. Albeverio S, Cognola, Spreafico M, Zerbini S. Singular perturbation with boundary conditions and the Casimir effect in the half space. J Math Phys. (2010) 51 . doi: 10.1063/1.3397551

2. Bytsenko AA, Elizalde E, Odintsov S, Romeo A, and Zerbini S. Zeta-function regularization with applications. World Sci. (1994).

3. Gilkey PB. Invariance theorems, the heat equation, and the Atiyah-Singer index theorem. Stud Adv Math. (1995).

4. Hawking SW. Zeta function regularization of path integral in curved spacetime. Comm. Math. Phys. (1977) 55:133-48.

5. Quine JR, Heydari SH, Song RY. Zeta regularized products. Trans Am Math Soc. (1993) 338:213-30.

6. Jorgenson J, Lang S. Basic analysis of regularized series and products. (1993) 1564.

7. Jorgenson J, Lang S. Explicit formulas for regularized series and products. (1994) 1593.

8. Müller W. Relative zeta functions, relative determinants and scattering theory. Commun Math Phys. (1998) 192:309-47.

9. Ray DB, Singer IM. R-torsion and the Laplacian on Riemannian manifolds. Adv Math. (1974) 7:145-210.

10. Rosenberg S. The Laplacian on a Riemannian manifold. An introduction to analysis on manifolds. (London Mathematical Society Student Texts). Sao Carlos: Cambridge University Press (1997).

11. Sarnak P. Determinants of laplacians. Comm Math Phys. (1987) 110:113-20.

12. Spreafico M. Zeta function and regularized determinant on a disc and on a cone. J Geom Phys. (2005) 54:355-371.

13. Spreafico $M$. Zeta invariants for sequences of spectral type, special functions and the Lerch formula. Proc R Soc. Edinb. (2006) 281:865-89. doi: $10.1017 /$ S0308210500004777

14. Spreafico M. Zeta invariants for Dirichlet series. Pac J Math. (2006) 274:185-99. doi: 10.2140/pim.2006.224.185

15. Spreafico M. Zeta invariants for double sequences of spectral type. Proc. Am. Math. Soc. (2012) 140:1881-69. doi: 10.2140/pjm.2006.224.185

16. Spreafico M, Zerbini S. Finite temperature quantum field theory on non compact domains and application to delta interactions. Rep Math Phys. (2009) 63:163-77. doi: 10.1016/S0034-4877(09)00011-1

17. Voros A. Spectral functions, special functions and the Selberg zeta function. Comm Math Phys. (1987) 110:439-65. doi: 10.1007/BF01212422

Conflict of Interest Statement: The author declares that the research was conducted in the absence of any commercial or financial relationships that could be construed as a potential conflict of interest.

Received: 15 September 2013; accepted: 07 November 2013; published online: 25 November 2013.

Citation: Spreafico M (2013) Zeta regularization of infinite products. Front. Physics 1:23. doi: $10.3389 / f p h y .2013 .00023$

This article was submitted to Mathematical Physics, a section of the journal Frontiers in Physics.

Copyright (0) 2013 Spreafico. This is an open-access article distributed under the terms of the Creative Commons Attribution License (CC BY). The use, distribution or reproduction in other forums is permitted, provided the original author(s) or licensor are credited and that the original publication in this journal is cited, in accordance with accepted academic practice. No use, distribution or reproduction is permitted which does not comply with these terms. 